- Erratum

\title{
Correction: The Effect of Lifestyle Changes on Blood Pressure Control among Hypertensive Patients
}

\author{
Myung Hwa Yang' ${ }^{1}$, Seo Young Kang' ${ }^{1}$ Jung Ah Lee', Young Sik Kim ${ }^{1, *}$, Eun Ju Sung ${ }^{2}$, Ka-Young Lee ${ }^{3}$, Jun-Su Kim³ \\ Han Jin $\mathrm{Oh}^{4}$, Hee Chul Kang ${ }^{5}$, Sang Yeoup Lee ${ }^{6}$ \\ 'Department of Family Medicine, Asan Medical Center, University of Ulsan College of Medicine, Seoul, Korea \\ ${ }^{2}$ Department of Family Medicine, Kangbuk Samsung Hospital, Sungkyunkwan University School of Medicine, Seoul, Korea \\ ${ }^{3}$ Department of Family Medicine, Inje University Busan Paik Hospital, Inje University College of Medicine, Busan, Korea \\ ${ }^{4}$ Department of Family Medicine, Vievisnamuh Hospital, Seoul, Korea \\ ${ }^{5}$ Department of Family Medicine, Yonsei University College of Medicine, Seoul, Korea \\ ${ }^{6}$ Department of Family Medicine, Pusan National University Yangsan Hospital, Pusan National University School of Medicine, Yangsan, Korea
}

https://doi.org/10.4082/kjfm.2017.38.4.173

Korean J Fam Med 2017;38:173-180

There was an error in the article, "The effect of lifestyle changes on blood pressure control among hypertensive patients ${ }^{1)}$." The authors found an error after the publication of this work ${ }^{1)}$ and asked the editorial board to correct it. So we would like to replace Table 3 of the original paper with a revised version. A revised version of Table 3 in the original article is given below.

We sincerely apologized to the readers.

\section{REFERENCE}

1. Yang MH, Kang SY, Lee JA, Kim YS, Sung EJ, Lee KY, et al. The effect of lifestyle changes on blood pressure control among hypertensive patients. Korean J Fam Med 2017;38:173-80. 
$<$ Original version Table 3>

Table 3. Changes in the performance index between patients with successful and failed blood pressure control

\begin{tabular}{|c|c|c|c|c|c|c|c|}
\hline \multirow{2}{*}{ Variable } & \multirow{2}{*}{ Total } & \multirow{2}{*}{ Failure } & \multirow{2}{*}{ Success } & \multicolumn{2}{|l|}{ Univariate $\mathrm{OR}$} & \multicolumn{2}{|l|}{ Adjusted $\mathrm{OR}^{*}$} \\
\hline & & & & $(95 \% \mathrm{Cl})$ & P-value & $(95 \% \mathrm{Cl})$ & P-value \\
\hline Reduced alcohol use among drinkers & 1,139 & & & & & & \\
\hline Yes & $88(7.7)$ & $22(25.0)$ & $66(75.0)$ & 1 (reference) & & 1 (reference) & \\
\hline No & $243(21.3)$ & $43(17.7)$ & $200(82.3)$ & $0.645(0.360-1.157)$ & 0.141 & $0.727(0.398-1.330)$ & 0.301 \\
\hline Unknown & $808(70.9)$ & & & & & & \\
\hline Reduction in frequency of alcohol consumption & 1,139 & & & & & & \\
\hline No change & $201(17.6)$ & $38(18.9)$ & $163(81.1)$ & 1 (reference) & & 1 (reference) & \\
\hline$<1 /$ wk & $24(2.1)$ & $2(8.3)$ & $22(91.7)$ & $0.390(0.088-1.730)$ & 0.215 & $0.421(0.093-1.895)$ & 0.260 \\
\hline $1-2 / w k$ & $14(1.2)$ & $2(14.3)$ & $12(85.7)$ & $0.715(0.154-3.328)$ & 0.669 & $0.569(0.119-2.713)$ & 0.479 \\
\hline$>3 /$ wk & $4(0.4)$ & $1(25.0)$ & $3(75.0)$ & $1.430(0.145-14.127)$ & 0.760 & $1.500(0.145-150494)$ & 0.734 \\
\hline Unknown $^{\dagger}$ & $896(78.7)$ & & & & & & \\
\hline Increased body mass index & 1,062 & & & & & & \\
\hline Yes & 250 & 59 (23.6) & $191(76.4)$ & 1 (reference) & & 1 (reference) & \\
\hline No & 812 & 101 (12.4) & $711(87.6)$ & 2.175 (1.519-3.113) & $<0.001$ & 2.010 (1.388-2.911) & $<0.001$ \\
\hline$>0.5 \mathrm{~kg} / \mathrm{m}^{2}$ & $96(9.0)$ & $25(26.0)$ & $71(74.0)$ & 1 (reference) & & 1 (reference) & \\
\hline$\leq 0.5 \mathrm{~kg} / \mathrm{m}^{2}$ & $966(91.0)$ & $135(14.0)$ & $831(86.0)$ & $2.044(1.420-2.942)$ & $<0.001$ & $2.189(1.309-3.659)$ & 0.003 \\
\hline Increased physical activity & 965 & & & & & & \\
\hline Yes & 882 & $128(14.5)$ & 754 (85.5) & 1 (reference) & & 1 (reference) & \\
\hline No & 83 & $21(25.3)$ & $62(74.7)$ & $1.995(1.175-3.387)$ & 0.011 & $2.377(1.351-4.183)$ & 0.003 \\
\hline Reduced salt intake & 1,096 & & & & & & \\
\hline Yes & 408 & $52(12.7)$ & $356(87.3)$ & 1 (reference) & & 1 (reference) & \\
\hline No & 688 & $121(17.6)$ & 567 (82.4) & 1.461 (1.029-2.075) & 0.034 & $1.324(0.914-1.917)$ & 0.138 \\
\hline
\end{tabular}

Values are presented as number or number (\%).

$\mathrm{OR}$, odds ratio; $\mathrm{Cl}$, confidence interval.

*Multivariate $\mathrm{OR}$ calculated by adjusting for age, sex, and comorbidity. ${ }^{+}$Persons with no classification.

$<$ Revised version Table 3>

Table 3. Changes in the performance index between patients with successful and failed blood pressure control

\begin{tabular}{|c|c|c|c|c|c|c|c|}
\hline \multirow{2}{*}{ Variable } & \multirow{2}{*}{ Total } & \multirow{2}{*}{ Failure } & \multirow{2}{*}{ Success } & \multicolumn{2}{|l|}{ Univariate $\mathrm{OR}$} & \multicolumn{2}{|l|}{ Adjusted $\mathrm{OR}^{*}$} \\
\hline & & & & $(95 \% \mathrm{Cl})$ & P-value & $(95 \% \mathrm{Cl})$ & P-value \\
\hline Reduced alcohol use among drinkers & 1,139 & & & & & & \\
\hline Yes & $88(7.7)$ & $22(25.0)$ & $66(75.0)$ & 1 (reference) & & 1 (reference) & \\
\hline No & $243(21.3)$ & $43(17.7)$ & $200(82.3)$ & $0.645(0.360-1.157)$ & 0.141 & $0.727(0.398-1.330)$ & 0.301 \\
\hline Unknown & $808(70.9)$ & & & & & & \\
\hline Reduction in frequency of alcohol consumption & 1,139 & & & & & & \\
\hline No change & $201(17.6)$ & $38(18.9)$ & $163(81.1)$ & 1 (reference) & & 1 (reference) & \\
\hline$<1 /$ wk & $24(2.1)$ & $2(8.3)$ & $22(91.7)$ & $0.390(0.088-1.730)$ & 0.215 & $0.421(0.093-1.895)$ & 0.260 \\
\hline $1-2 /$ wk & $14(1.2)$ & $2(14.3)$ & $12(85.7)$ & $0.715(0.154-3.328)$ & 0.669 & $0.569(0.119-2.713)$ & 0.479 \\
\hline$>3 /$ wk & $4(0.4)$ & $1(25.0)$ & $3(75.0)$ & $1.430(0.145-14.127)$ & 0.760 & $1.500(0.145-15.494)$ & 0.734 \\
\hline Unknown ${ }^{\dagger}$ & $896(78.7)$ & & & & & & \\
\hline Increased body mass index & 1,062 & & & & & & \\
\hline No & 812 & $101(12.4)$ & $711(87.6)$ & 1 (reference) & & 1 (reference) & \\
\hline Yes & 250 & $59(23.6)$ & $191(76.4)$ & $2.175(1.519-3.113)$ & $<0.001$ & $2.010(1.388-2.911)$ & $<0.001$ \\
\hline$>0.5 \mathrm{~kg} / \mathrm{m}^{2}$ & $966(91.0)$ & $135(14.0)$ & $831(86.0)$ & 1 (reference) & & 1 (reference) & \\
\hline$\leq 0.5 \mathrm{~kg} / \mathrm{m}^{2}$ & $96(9.0)$ & $25(26.0)$ & $71(74.0)$ & $2.044(1.420-2.942)$ & $<0.001$ & $2.189(1.309-3.659)$ & 0.003 \\
\hline Increased physical activity & 965 & & & & & & \\
\hline Yes & 882 & $128(14.5)$ & $754(85.5)$ & 1 (reference) & & 1 (reference) & \\
\hline No & 83 & $21(25.3)$ & $62(74.7)$ & $1.995(1.175-3.387)$ & 0.011 & $2.377(1.351-4.183)$ & 0.003 \\
\hline Reduced salt intake & 1,096 & & & & & & \\
\hline Yes & 408 & $52(12.7)$ & $356(87.3)$ & 1 (reference) & & 1 (reference) & \\
\hline No & 688 & $121(17.6)$ & 567 (82.4) & 1.461 (1.029-2.075) & 0.034 & $1.324(0.914-1.917)$ & 0.138 \\
\hline
\end{tabular}

Values are presented as number or number (\%).

$\mathrm{OR}$, odds ratio; $\mathrm{Cl}$, confidence interval.

*Multivariate OR calculated by adjusting for age, sex, and comorbidity. ${ }^{+}$Persons with no classification. 